\title{
On Two Extension Formulas for Lauricella's Function of the Second Kind of Several Variables
}

\author{
Ahmed Ali Atash', Ahmed Ali Al-Gonah ${ }^{2}$ \\ ${ }^{1}$ Department of Mathematics, Faculty of Education-Shabwah, Aden University, Aden, Yemen \\ ${ }^{2}$ Department of Mathematics, Faculty of Education-Aden, Aden University, Aden, Yemen \\ Email: ah-a-atash@hotmail.com,gonah1977@yahoo.com
}

Received 1 February 2016; accepted 27 March 2016; published 30 March 2016

Copyright (C) 2016 by authors and Scientific Research Publishing Inc.

This work is licensed under the Creative Commons Attribution International License (CC BY).

http://creativecommons.org/licenses/by/4.0/

(c) (i)

\section{Abstract}

The aim of this research paper is to derive two extension formulas for Lauricella's function of the second kind of several variables with the help of generalized Dixon's theorem on the sum of the series ${ }_{3} F_{2}(1)$ obtained by Lavoie et al. [1]. Some special cases of these formulas are also deduced.

\section{Keywords}

\section{Extension Formulas, Lauricella's Function, Dixon's Theorem, Hypergeometric Functions}

\section{Introduction}

The Lauricella's function $F_{B}^{(r)}$ is defined and represented as follows [2]

$$
\begin{gathered}
F_{B}^{(r)}\left(a_{1}, \cdots, a_{r}, b_{1}, \cdots, b_{r} ; c ; x_{1}, \cdots, x_{r}\right) \\
=\sum_{m_{1}, \cdots, m_{r}=0}^{\infty} \frac{\left(a_{1}\right)_{m_{1}} \cdots\left(a_{r}\right)_{m_{r}}\left(b_{1}\right)_{m_{1}} \cdots\left(b_{r}\right)_{m_{r}}}{(c)_{m_{1}+\cdots+m_{r}}} \frac{x_{1}^{m_{1}}}{m_{1} !} \cdots \frac{x_{r}^{m_{r}}}{m_{r} !} \\
\max \left\{\left|x_{1}\right|, \cdots,\left|x_{r}\right|\right\}<1 ;
\end{gathered}
$$

where $(a)_{n}$ denotes the Pochhammer's symbol defined by

How to cite this paper: Atash, A.A. and Al-Gonah, A.A. (2016) On Two Extension Formulas for Lauricella's Function of the Second Kind of Several Variables. Journal of Applied Mathematics and Physics, 4, 571-577. 


$$
\begin{gathered}
(a)_{n}= \begin{cases}1, & \text { if } n=0 \\
a(a+1)(a+2) \cdots(a+n-1), & \text { if } n=1,2,3, \cdots\end{cases} \\
=\frac{\Gamma(a+n)}{\Gamma(a)}, \quad a \neq 0,-1,-2, \cdots
\end{gathered}
$$

Also, we note that

$$
\begin{gathered}
\Gamma\left(\frac{1}{2}\right) \Gamma(1+a)=2^{a} \Gamma\left(\frac{1}{2}+\frac{1}{2} a\right) \Gamma\left(1+\frac{1}{2} a\right) \\
(a)_{2 n}=2^{2 n}\left(\frac{1}{2} a\right)_{n}\left(\frac{1}{2} a+\frac{1}{2}\right)_{n} \\
\frac{\Gamma(a-n)}{\Gamma(a)}=\frac{(-1)^{n}}{(1-a)_{n}} \\
(2 n) !=2^{2 n}\left(\frac{1}{2}\right)_{n} n !
\end{gathered}
$$

The generalized Lauricella's function of several variables is defined as follows [2]

$$
\begin{aligned}
& \left.F \begin{array}{l}
A: B^{\prime} ; \cdots ; B^{(n)} \\
C: D^{\prime} ; \cdots ; D^{(n)}
\end{array} z_{1}, \cdots, z_{n}\right] \\
& \left.\equiv F \begin{array}{c}
A: B^{\prime} ; \cdots ; B^{(n)} \\
C: D^{\prime} ; \cdots ; D^{(n)}\left(\left[(a): \theta^{\prime}, \cdots, \theta^{(n)}\right]:\left[\left(b^{\prime}\right): \phi^{\prime}\right] ; \cdots ;\left[\left(b^{(n)}\right): \phi^{(n)}\right] ;\right. \\
{\left[(c): \psi^{\prime}, \cdots, \psi^{(n)}\right]:\left[\left(d^{\prime}\right): \delta^{\prime}\right] ; \cdots ;\left[\left(d^{(n)}\right): \delta^{(n)}\right] ;}
\end{array}\right) \\
& =\sum_{m_{1}, \cdots, m_{n}=0}^{\infty} \Omega\left(m_{1}, \cdots, m_{n}\right) \frac{z_{1}^{m_{1}}}{m_{1} !} \cdots \frac{z_{n}^{m_{n}}}{m_{n} !},
\end{aligned}
$$

where

$$
\Omega\left(m_{1}, \cdots, m_{n}\right)=\frac{\prod_{j=1}^{A}\left(a_{j}\right)_{m_{1} \theta_{j}^{\prime}+\cdots+m_{n} \theta_{j}^{(n)}} \prod_{j=1}^{B^{\prime}}\left(b_{j}^{\prime}\right)_{m_{1} \phi_{j}^{\prime}} \cdots \prod_{j=1}^{B}\left(b_{j}^{(n)}\right)_{m_{n} \phi_{j}^{(n)}}}{\prod_{j=1}^{C}\left(c_{j}\right)_{m_{1} \psi_{j}^{\prime}+\cdots+m_{n} \psi_{j}^{(n)}} \prod_{j=1}^{D^{\prime}}\left(d_{j}^{\prime}\right)_{m_{1} \delta_{j}^{\prime}} \ldots \prod_{j=1}^{D^{(n)}}\left(d_{j}^{(n)}\right)_{m_{n} \delta_{j}^{(n)}}}
$$

the coefficients $\theta_{j}^{(k)}, j=1,2 \cdots, A ; \phi_{j}^{(k)}, j=1,2, \cdots, B^{(k)} ; \psi_{j}^{(k)}, j=1,2, \cdots, C ; \delta_{j}^{(k)}, j=1,2, \cdots, D^{(k)}$, for all $k \in\{1,2, \cdots, n\}$ are real and positive; $(a)$ abbreviates the array of $A$ parameters; $a_{1}, \cdots a_{A},\left(b^{(k)}\right)$ abbreviate the array of $B^{(k)}$ parameters $b_{j}^{(k)}, j=1,2, \cdots, B^{(k)}$ for all $k \in\{1,2, \cdots, n\}$ with similar inter pretations for $(c)$ and $\left(d^{(k)}\right) \quad k \in 1,2, \cdots, n$; et cetera . Note that, when the coefficients in Equation (1.8) equal to 1, the generalized Lauricella function (1.8) reduces to the following multivariable extension of the Kamp'e de F'eriet function [2]:

$$
\begin{aligned}
F_{l: m_{1} ; \cdots ; m_{n}}^{p: q_{1} ; \cdots ; q_{n}}\left[z_{1}, \cdots, z_{n}\right] & \left.\equiv F \begin{array}{l}
p: q_{1} ; \cdots ; q_{n}( \\
l: m_{1} ; \cdots ; m_{n}\left(a_{p}\right):\left(b_{q_{1}}^{\prime}\right) ; \cdots ;\left(b_{q_{n}}^{(n)}\right) ; \\
\left(c_{l}\right):\left(d_{m_{1}}^{\prime}\right) ; \cdots ;\left(d_{m_{n}}^{(n)}\right) ; z_{1}, \cdots, z_{n}
\end{array}\right) \\
& =\sum_{s_{1}, \cdots, s_{n}=0}^{\infty} \Omega\left(s_{1}, \cdots, s_{n}\right) \frac{z_{1}^{s_{1}}}{s_{1} !} \cdots \frac{z_{n}^{s_{n}}}{s_{n} !}
\end{aligned}
$$


where

$$
\Omega\left(s_{1}, \cdots, s_{n}\right)=\frac{\prod_{j=1}^{p}\left(a_{j}\right)_{s_{1}+\cdots+s_{n}} \prod_{j=1}^{q_{1}}\left(b_{j}^{\prime}\right)_{s_{1}} \cdots \prod_{j=1}^{q_{n}}\left(b_{j}^{(n)}\right)_{s_{n}}}{\prod_{j=1}^{l}\left(c_{j}\right)_{s_{1}+\cdots+s_{n}} \prod_{j=1}^{m_{1}}\left(d_{j}^{\prime}\right)_{s_{1}} \cdots \prod_{j=1}^{m_{n}}\left(d_{j}^{(n)}\right)_{s_{n}}} .
$$

In the theory of hypergeometric series, classical summation theorems such as Dixon, Watson and Whipple for the series ${ }_{3} F_{2}$, have many generalizations and wide applications; see for example [1] [3]-[6]. In the present investigation, we shall require the following generalization of the classical Dixon's theorem for the series ${ }_{3} F_{2}(1)$ [1]:

$$
\begin{aligned}
& { }_{3} F_{2}\left[\begin{array}{c}
a, b, c \\
1+a-b+i, 1+a-c+i+j ;
\end{array}\right] \\
& =\frac{2^{-2 c+i+j} \Gamma(1+a-b+i) \Gamma(1+a-c+i+j) \Gamma\left(b-\frac{1}{2}|i|-\frac{1}{2} i\right) \Gamma\left(c-\frac{1}{2}(i+j+|i+j|)\right)}{\Gamma(b) \Gamma(c) \Gamma(1+a-2 c+i+j) \Gamma(1+a-b-c+i+j)} \\
& \times\left\{\begin{array}{c}
A_{i, j} \frac{\Gamma\left(\frac{1}{2} a-c+\frac{1}{2}+\left[\frac{i+j+1}{2}\right]\right) \Gamma\left(\frac{1}{2} a-b-c+1+i+\left[\frac{j+1}{2}\right]\right)}{\Gamma\left(\frac{1}{2} a+\frac{1}{2}\right) \Gamma\left(\frac{1}{2} a-b+1+\left[\frac{i}{2}\right]\right)} \\
+B_{i, j} \frac{\Gamma\left(\frac{1}{2} a-c+1+\left[\frac{i+j}{2}\right]\right) \Gamma\left(\frac{1}{2} a-b-c+\frac{3}{2}+i+\left[\frac{j}{2}\right]\right)}{\Gamma\left(\frac{1}{2} a\right) \Gamma\left(\frac{1}{2} a-b+\frac{1}{2}+\left[\frac{i+1}{2}\right]\right)} \\
(R(a-2 b-2 c)>-2-2 i-j ;
\end{array},\right. \\
& i=-3,-2,-1,0,1,2 ; j=0,1,2,3)
\end{aligned}
$$

where $[x]$ denotes the greatest integer less than or equal to $x$ and $|x|$ denotes the usual absolute value of $x$. The coefficients $A_{i, j}$ and $B_{i, j}$ are given respectively in [1]. When $i=j=0,(1.12)$ reduces immediately to the classical Dixon's theorem [3], (see also [6])

$$
\begin{gathered}
{ }_{3} F_{2}\left[\begin{array}{c}
a, b, c \\
1+a-b, 1+a-c ;
\end{array}\right] \\
=\frac{\Gamma\left(1+\frac{1}{2} a\right) \Gamma(1+a-b) \Gamma(1+a-c) \Gamma\left(1+\frac{1}{2} a-b-c\right)}{\Gamma(1+a) \Gamma\left(1+\frac{1}{2} a-b\right) \Gamma\left(1+\frac{1}{2} a-c\right) \Gamma(1+a-b-c)} \\
\{R(a-2 b-2 c)>-2\} .
\end{gathered}
$$

\section{Extension Formulas}

In this section, the following two extension formulas for Lauricella's function of the second kind of several variables will be established: 


$$
\begin{aligned}
& F_{B}^{(2 r)}\left(a_{1}-i, a_{1}, \cdots, a_{r}-i, a_{r}, b_{1}-i-j, b_{1}, \cdots, b_{r}-i-j, b_{r} ; c ; x_{1},-x_{1}, \cdots, x_{r},-x_{r}\right) \\
& =\sum_{m_{1}=0}^{\infty} \cdots \sum_{m_{r}=0}^{\infty} \frac{\left(a_{1}-i\right)_{2 m_{1}} \cdots\left(a_{r}-i\right)_{2 m_{r}}\left(b_{1}-i-j\right)_{2 m_{1}} \cdots\left(b_{r}-i-j\right)_{2 m_{r}} x_{1}^{2 m_{1}} \cdots x_{r}^{2 m_{r}}}{(c)_{2 m_{1}+\cdots+2 m_{r}}\left(2 m_{1}\right) ! \cdots\left(2 m_{r}\right) !} \\
& \times I_{1}\left(a_{1}, b_{1}, 2 m_{1}, i, j\right)\left\{A_{i, j}^{(1)} A_{1}\left(a_{1}, b_{1}, 2 m_{1}, i, j\right)+B_{i, j}^{(1)} B_{1}\left(a_{1}, b_{1}, 2 m_{1}, i, j\right)\right\} \times \cdots \\
& \times I_{r}\left(a_{r}, b_{r}, 2 m_{r}, i, j\right)\left\{A_{i, j}^{(r)} A_{r}\left(a_{r}, b_{r}, 2 m_{r}, i, j\right)+B_{i, j}^{(r)} B_{r}\left(a_{r}, b_{r}, 2 m_{r}, i, j\right)\right\}+\cdots \\
& +\sum_{m_{1}=0}^{\infty} \cdots \sum_{m_{r}=0}^{\infty} \frac{\left(a_{1}-i\right)_{2 m_{1}+1} \cdots\left(a_{r}-i\right)_{2 m_{r}+1}\left(b_{1}-i-j\right)_{2 m_{1}+1} \cdots\left(b_{r}-i-j\right)_{2 m_{r}+1} x_{1}^{2 m_{1}+1} \cdots x_{r}^{2 m_{r}+1}}{(c)_{2 m_{1}+1+\cdots+2 m_{r}+1}\left(2 m_{1}+1\right) ! \cdots\left(2 m_{r}+1\right) !} \\
& \times I_{1}\left(a_{1}, b_{1}, 2 m_{1}+1, i, j\right)\left\{C_{i, j}^{(1)} A_{1}\left(a_{1}, b_{1}, 2 m_{1}+1, i, j\right)+D_{i, j}^{(1)} B_{1}\left(a_{1}, b_{1}, 2 m_{1}+1, i, j\right)\right\} \times \cdots \\
& \times I_{r}\left(a_{r}, b_{r}, 2 m_{r}+1, i, j\right)\left\{C_{i, j}^{(r)} A_{r}\left(a_{r}, b_{r}, 2 m_{r}+1, i, j\right)+D_{i, j}^{(r)} B_{r}\left(a_{r}, b_{r}, 2 m_{r}+1, i, j\right)\right\}
\end{aligned}
$$

and

$$
\begin{aligned}
& F_{B}^{(2 r+1)}\left(a, a_{1}-i, a_{1}, \cdots, a_{r}-i, a_{r}, b, b_{1}-i-j, b_{1}, \cdots, b_{r}-i-j, b_{r} ; c ; x, x_{1},-x_{1}, \cdots, x_{r},-x_{r}\right) \\
& =\sum_{m=0}^{\infty} \sum_{m_{1}=0}^{\infty} \cdots \sum_{m_{r}=0}^{\infty} \frac{(a)_{m}\left(a_{1}-i\right)_{2 m_{1}} \cdots\left(a_{r}-i\right)_{2 m_{r}}(b)_{m}\left(b_{1}-i-j\right)_{2 m_{1}} \cdots\left(b_{r}-i-j\right)_{2 m_{r}} x^{m} x_{1}^{2 m_{1}} \cdots x_{r}^{2 m_{r}}}{(c)_{m+2 m_{1}+\cdots+2 m_{r}} m !\left(2 m_{1}\right) ! \cdots\left(2 m_{r}\right) !} \\
& \times I_{1}\left(a_{1}, b_{1}, 2 m_{1}, i, j\right)\left\{A_{i, j}^{(1)} A_{1}\left(a_{1}, b_{1}, 2 m_{1}, i, j\right)+B_{i, j}^{(1)} B_{1}\left(a_{1}, b_{1}, 2 m_{1}, i, j\right)\right\} \times \cdots \\
& \times I_{r}\left(a_{r}, b_{r}, 2 m_{r}, i, j\right)\left\{A_{i, j}^{(r)} A_{r}\left(a_{r}, b_{r}, 2 m_{r}, i, j\right)+B_{i, j}^{(r)} B_{r}\left(a_{r}, b_{r}, 2 m_{r}, i, j\right)\right\}+\cdots \\
& +\sum_{m=0}^{\infty} \sum_{m_{1}=0}^{\infty} \cdots \sum_{m_{r}=0}^{\infty} \frac{(a)_{m}\left(a_{1}-i\right)_{2 m_{1}+1} \cdots\left(a_{r}-i\right)_{2 m_{r}+1}}{(c)_{m+2 m_{1}+1+\cdots+2 m_{r}+1}} \\
& \times \frac{(b)_{m}\left(b_{1}-i-j\right)_{2 m_{1}+1} \cdots\left(b_{r}-i-j\right)_{2 m_{r}+1} x^{m} x_{1}^{2 m_{1}+1} \cdots x_{r}^{2 m_{r}+1}}{m !\left(2 m_{1}+1\right) ! \cdots\left(2 m_{r}+1\right) !} \\
& \times I_{1}\left(a_{1}, b_{1}, 2 m_{1}+1, i, j\right)\left\{C_{i, j}^{(1)} A_{1}\left(a_{1}, b_{1}, 2 m_{1}+1, i, j\right)+D_{i, j}^{(1)} B_{1}\left(a_{1}, b_{1}, 2 m_{1}+1, i, j\right)\right\} \times \cdots \\
& \times I_{r}\left(a_{r}, b_{r}, 2 m_{r}+1, i, j\right)\left\{C_{i, j}^{(r)} A_{r}\left(a_{r}, b_{r}, 2 m_{r}+1, i, j\right)+D_{i, j}^{(r)} B_{r}\left(a_{r}, b_{r}, 2 m_{r}+1, i, j\right)\right\}
\end{aligned}
$$

where

$$
\begin{aligned}
I_{r}\left(a_{r}, b_{r}, m_{r}, i, j\right)= & 2^{-2 b_{r}+i+j} \Gamma\left(1-m_{r}-a_{r}+i\right) \Gamma\left(1-m_{r}-b_{r}+i+j\right) \\
& \times \frac{\Gamma\left(a_{r}-\frac{1}{2}|i|-\frac{1}{2} i\right) \Gamma\left(b_{r}-\frac{1}{2}(i+j+|i+j|)\right)}{\Gamma\left(a_{r}\right) \Gamma\left(b_{r}\right) \Gamma\left(1-m_{r}-2 b_{r}+i+j\right) \Gamma\left(1-m_{r}-a_{r}-b_{r}+i+j\right)} \\
A_{r}\left(a_{r}, b_{r}, m_{r}, i, j\right)= & \frac{\Gamma\left(\frac{1}{2}-\frac{1}{2} m_{r}-b_{r}+\left[\frac{i+j+1}{2}\right]\right) \Gamma\left(1-\frac{1}{2} m_{r}-a_{r}-b_{r}+i+\left[\frac{j+1}{2}\right]\right)}{\Gamma\left(\frac{1}{2}-\frac{1}{2} m_{r}\right) \Gamma\left(1-\frac{1}{2} m_{r}-a_{r}+\left[\frac{i}{2}\right]\right)}
\end{aligned}
$$




$$
B_{r}\left(a_{r}, b_{r}, m_{r}, i, j\right)=\frac{\Gamma\left(1-\frac{1}{2} m_{r}-b_{r}+\left[\frac{i+j}{2}\right]\right) \Gamma\left(\frac{3}{2}-\frac{1}{2} m_{r}-a_{r}-b_{r}+i+\left[\frac{j}{2}\right]\right)}{\Gamma\left(-\frac{1}{2} m_{r}\right) \Gamma\left(\frac{1}{2}-\frac{1}{2} m_{r}-a_{r}+\left[\frac{i+1}{2}\right]\right)}
$$

for $i=-3,-2,-1,0,1,2 ; j=0,1,2,3 ; r=1,2,3, \cdots$.

The coefficients $A_{i, j}^{(r)}, B_{i, j}^{(r)}, C_{i, j}^{(r)}$ and $D_{i, j}^{(r)}$ can be obtained from the tables of $A_{i, j}$ and $B_{i, j}$ given in [1] by replacing $a$ by $-2 m_{r}$ and $-2 m_{r}-1$ respectively.

Proof of (2.1): Denoting the left hand side of (2.1) by S, expanding $F_{B}^{(2 r)}$ in a power series and using the results [2]:

$$
\begin{gathered}
(a)_{m+n}=(a)_{m}(a+m)_{n} \\
\sum_{m=0}^{\infty} \sum_{n=0}^{\infty} A(n, m)=\sum_{m=0}^{\infty} \sum_{n=0}^{m} A(n, m-n) \\
(a)_{m-n}=\frac{(-1)^{n}(a)_{m}}{(1-a-m)_{n}}, 0 \leq n \leq m \text { and }(m-n) !=\frac{(-1)^{n} m !}{(-m)_{n}}, 0 \leq n \leq m,
\end{gathered}
$$

we get

$$
\begin{aligned}
S & =\sum_{m_{1}=0}^{\infty} \cdots \sum_{m_{r}=0}^{\infty} \frac{\left(a_{1}-i\right)_{m_{1}} \cdots\left(a_{r}-i\right)_{m_{r}}\left(b_{1}-i-j\right)_{m_{1}} \cdots\left(b_{r}-i-j\right)_{m_{r}} x_{1}^{m_{1}} \cdots x_{r}^{m_{r}}}{(c)_{m_{1}+\cdots+m_{r}} m_{1} ! \cdots m_{r} !} \\
& \times f_{1}\left(a_{1}, b_{1}, m_{1}, i, j\right) \times \cdots \times f_{r}\left(a_{r}, b_{r}, m_{r}, i, j\right)
\end{aligned}
$$

where

$$
f_{r}\left(a_{r}, b_{r}, m_{r}, i, j\right)={ }_{3} F_{2}\left[\begin{array}{cc}
-m_{r}, a_{r}, b_{r} & ; \\
1-m_{r}-a_{r}+i, 1-m_{r}-b_{r}+i+j ;
\end{array}\right] .
$$

Separating (2.9) into its even and odd terms, we have

$$
\begin{aligned}
S & =\sum_{m_{1}=0}^{\infty} \cdots \sum_{m_{r}=0}^{\infty} \frac{\left(a_{1}-i\right)_{2 m_{1}} \cdots\left(a_{r}-i\right)_{2 m_{r}}\left(b_{1}-i-j\right)_{2 m_{1}} \cdots\left(b_{r}-i-j\right)_{2 m_{r}} x_{1}^{2 m_{1}} \cdots x_{r}^{2 m_{r}}}{(c)_{2 m_{1}+\cdots+2 m_{r}} 2 m_{1} ! \cdots 2 m_{r} !} \\
& \times f_{1}\left(a_{1}, b_{1}, 2 m_{1}, i, j\right) \times \cdots \times f_{r}\left(a_{r}, b_{r}, 2 m_{r}, i, j\right)+\sum_{m_{1}=0}^{\infty} \cdots \sum_{m_{r}=0}^{\infty} \frac{\left(a_{1}-i\right)_{2 m_{1}+1}\left(a_{2}-i\right)_{2 m_{2}} \cdots\left(a_{r}-i\right)_{2 m_{r}}}{(c)_{2 m_{1}+1+2 m_{2}+\cdots+2 m_{r}}} \\
& \times \frac{\left(b_{1}-i-j\right)_{2 m_{1}+1}\left(b_{2}-i-j\right)_{2 m_{2}} \cdots\left(b_{r}-i-j\right)_{2 m_{r}} x_{1}^{2 m_{1}+1} x^{2 m_{2}} \cdots x_{r}^{2 m_{r}}}{\left(2 m_{1}+1\right) !\left(2 m_{2}\right) ! \cdots\left(2 m_{r}\right) !} \times f_{1}\left(a_{1}, b_{1}, 2 m_{1}+1, i, j\right) \\
& \times f_{2}\left(a_{2}, b_{2}, 2 m_{2}, i, j\right) \times \cdots \times f_{r}\left(a_{r}, b_{r}, 2 m_{r}, i, j\right)+\cdots+\sum_{m_{1}=0}^{\infty} \cdots \sum_{m_{r}=0}^{\infty} \frac{\left(a_{1}-i\right)_{2 m_{1}} \cdots\left(a_{r-1}-i\right)_{2 m_{r-1}}\left(a_{r}-i\right)_{2 m_{r}+1}}{(a)_{2 m_{1}+\cdots+2 m_{r-1}+2 m_{r}+1}} \\
& \times \frac{\left(b_{1}-i-j\right)_{2 m_{1}} \cdots\left(b_{r-1}-i-j\right)_{2 m_{r-1}}\left(b_{r}-i-j\right)_{2 m_{r}+1} x_{1}^{2 m_{1}} \cdots x_{r-1}^{2 m_{r-1}} x_{r}^{2 m_{r}+1}}{\left(2 m_{1}\right) ! \cdots\left(2 m_{r-1}\right) !\left(2 m_{r}+1\right) !} \\
& \times f_{1}\left(a_{1}, b_{1}, 2 m_{1}, i, j\right) \times \cdots \times f_{r-1}\left(a_{r-1}, b_{r-1}, 2 m_{r-1}, i, j\right) \times f_{r}\left(a_{r}, b_{r}, 2 m_{r}+1, i, j\right) \\
& +\sum_{m_{1}=0}^{\infty} \cdots \sum_{m_{r}=0}^{\infty} \frac{\left(a_{1}-i\right)_{2 m_{1}+1} \cdots\left(a_{r}-i\right)_{2 m_{r}+1}\left(b_{1}-i\right)_{2 m_{1}+1}\left(b_{r}-i\right)_{2 m_{r}+1} x_{1}^{2 m_{1}+1} \cdots x_{r}^{2 m_{r}+1}}{(c)_{2 m_{1}+1+\cdots+2 m_{r}+1}\left(2 m_{1}+1\right) ! \cdots\left(2 m_{r}+1\right) !} \\
& \times f_{1}\left(a_{1}, b_{1}, 2 m_{1}+1, i, j\right) \times \cdots \times f_{r}\left(a_{r}, b_{r}, 2 m_{r}+1, i, j\right)
\end{aligned}
$$


Finally, in (2.11) if we use the result (1.12), then we obtain the right hand side of (2.1). This completes the proof of (2.1). The result (2.2) can be proved by the similar manner.

\section{Special Cases}

1) In (2.1), if we take $i=j=0$ and use the results (1.3)-(1.7), then after some simplification we obtain the following transformation formula:

$$
\begin{aligned}
& F_{B}^{(2 r)}\left(a_{1}, a_{1}, \cdots, a_{r}, a_{r}, b_{1}, b_{1}, \cdots, b_{r}, b_{r} ; c ; x_{1},-x_{1}, x_{2},-x_{2}, \cdots, x_{r},-x_{r}\right) \\
& =F \begin{array}{c}
0: 4 ; \cdots ; 4 \\
2: 1 ; \cdots ; 1
\end{array}\left[\frac{1}{2} c, \frac{1}{2} c+\frac{1}{2}: a_{1}, b_{1}, \frac{1}{2}\left(a_{1}+b_{1}\right), \frac{1}{2}\left(a_{1}+b_{1}+1\right) ;\right. \\
& \left.\cdots ; a_{r}, b_{r}, \frac{1}{2}\left(a_{r}+b_{r}\right) \frac{1}{2}\left(a_{r}+b_{r}+1\right) ; x_{1}^{2}, \cdots, x_{r}^{2}\right]
\end{aligned}
$$

which for $r=1$, reduces immediately to a known result of Bailey [7]

$$
F_{3}[a, a, b, b ; c ; x,-x]={ }_{4} F_{3}\left[\begin{array}{c}
a, b, \frac{1}{2}(a+b), \frac{1}{2}(a+b+1) ; x^{2} \\
\frac{1}{2} c, \frac{1}{2} c+\frac{1}{2}, a+b ;
\end{array}\right]
$$

where $F_{3}$ is Appell's function [2].

2) Similarly, in (2.2) if we take $i=j=0$ and use the results (1.3)-(1.7), then we obtain the following transformation formula:

$$
\begin{aligned}
& F_{B}^{(2 r+1)}\left(a, a_{1}, a_{1}, \cdots, a_{r}, a_{r}, b, b_{1}, b_{1}, \cdots, b_{r}, b_{r} ; c ; x, x_{1},-x_{1}, \cdots, x_{r},-x_{r}\right) \\
& =F \begin{array}{c}
0: 2 ; 4 ; \cdots ; 4 \\
1: 0 ; 1 ; \cdots ; 1
\end{array}\left[\begin{array}{c}
----(a: 1),(b: 1) ;\left(a_{1}: 1\right),\left(b_{1}: 1\right),\left(\frac{1}{2}\left(a_{1}+b_{1}\right): 1\right),\left(\frac{1}{2}\left(a_{1}+b_{1}+1\right): 1\right) ; \\
(c: 1,2, \cdots, 2):----;
\end{array}\right. \\
& \begin{array}{l}
\cdots ;\left(a_{r}: 1\right),\left(b_{r}: 1\right),\left(\frac{1}{2}\left(a_{r}+b_{r}\right): 1\right),\left(\frac{1}{2}\left(a_{r}+b_{r}+1\right): 1\right) ; x, 4 x_{1}^{2}, \cdots, 4 x_{r}^{2} \\
\cdots ; \\
\left(a_{r}+b_{r}: 1\right)
\end{array}
\end{aligned}
$$

3) In (2.2) if we take $r=1$, then we get a known extension formulas [8] for Lauricella's function of three variables $F_{B}^{(3)}\left(a, a_{1}-i, a_{1}, b, b_{1}-i-j, b_{1} ; c ; x, x_{1},-x_{1}\right)$ for $\{i=-3,-2,-1,0,1,2 ; j=0,1,2,3\}$.

\section{References}

[1] Lavoie, J.L., Grondin, F., Rathie, A.K. and Arora, K. (1994) Generalizations of Dixon's Theorem on the Sum of a ${ }_{3} F_{2}$. Mathematics of Computation, 62, 267-276.

[2] Srivastava, H.M. and Manocha, H.L. (1984) A Treatise on Generating Functions. Hasted Press, New York.

[3] Bailey, W.N. (1935) Generalized Hypergeometric Series. Cambridge University Press, Cambridge.

[4] Lavoie, J.L., Grondin, F. and Rathie, A.K. (1992) Generalizations of Watson's Theorem on the Sum of a ${ }_{3} F_{2}$. Indian Journal of Mathematics, 34, 23-32.

[5] Lavoie, J.L., Grondin, F. and Rathie, A.K. (1996) Generalizations of Whipple's Theorem on the Sum of a ${ }_{3} F_{2}$. Journal of Computational and Applied Mathematics, 72, 293-300. http://dx.doi.org/10.1016/0377-0427(95)00279-0

[6] Rainville, E.D. (1960) Special Functions. The Macmillan Company, New York. 
[7] Bailey, W.N. (1953) On the Sum of Terminating ${ }_{3} F_{2}(1)$. Quarterly Journal of Mathematics Oxford, 2, 237-240. http://dx.doi.org/10.1093/qmath/4.1.237

[8] Atash, A.A. (2015) An Extension Formulas of Lauricella's Functions by Applications of Dixon's Summation Theorem. Applications and Applied Mathematics, 10, 1007-1018. 